\title{
Generalized order and related growth measure of composite entire function of several complex variables on the basis of central index
}

\author{
Dilip Chandra Pramanik ${ }^{1}$, Manab Biswas ${ }^{2}$ and Kapil Roy ${ }^{3}$ \\ ${ }^{1}$ Department of Mathematics, University of North Bengal, Raja Rammohanpur, Dist-Darjeeling, West Bengal, India \\ ${ }^{2}$ Barabilla High School, P.O. Haptiagach Dist-Uttar Dinajpur, West Bengal, India \\ ${ }^{3}$ Department of Mathematics, University of North Bengal,India
}

Received: 26 August 2017, Accepted: 17 May 2018

Published online: 6 August 2018.

\begin{abstract}
In this paper, we define generalized order of an entire function of several complex variables in terms of central index and use it to estimate the growth properties of composite entire function of several complex variables with respect to one of the factor of the composition function.
\end{abstract}

Keywords: Entire function, maximum modulus, maximum term, central index, generalized order (generalized lower order).

\section{Introduction, Definitions and Notations}

We denote complex $n$-space by $\mathbb{C}^{n}$ and indicate its elements (points):

$$
\left(z_{1}, z_{2}, \ldots, z_{n}\right),\left(\left|z_{1}\right|,\left|z_{2}\right|, \ldots,\left|z_{n}\right|\right),\left(r_{1}, r_{2}, \ldots, r_{n}\right),\left(k_{1}, k_{2}, \ldots, k_{n}\right)
$$

by their corresponding symbols $z,|z|, r, k$ etc. Throughout $\Omega=\Omega_{n}$ stands for a nonempty open complete $n$-circular region in $C^{n}$ (see $\S 3.3$ of [3]) with center at $(0,0, \ldots, 0)$, the zero element of $\mathbb{C}^{n}$. We write

$$
|\Omega|=\{r: r=|z| \text { for some } z \in \Omega\}
$$

and

$$
\Omega^{+}=\left\{r: r \in|\Omega|, \text { no } r_{j}=0,1 \leq j \leq n\right\}
$$

and regard these as subsets of the $n$-dimensional Euclidean space $\mathbb{R}^{n}$. For any $r, s \in \mathbb{R}^{n}$, we say that

(i) $r \leq s$ or $s \geq r$, if and only if $r_{j} \leq s_{j}$ for $1 \leq j \leq n$,

(ii) $r<s$ or $s>r$, if and only if $r \leq s$ but $r$ is not equal to $s$

and

(iii) $r<<s$ or $s>>r$, if and only if $r_{j}<s_{j}$ for $1 \leq j \leq n$.

A function $f(z), z \in \mathbb{C}^{n}$ is said to be analytic at a point $\xi \in \mathbb{C}^{n}$ if it can be expanded in some neighborhood of $\xi$ as an absolutely convergent power series. If we assume $\xi=(0,0, \ldots, 0)$, then $f(z)$ has representation(see [6] and [8]).

$$
f(z)=\sum_{k=(0,0, \ldots, 0)}^{\infty} a_{k_{1}, k_{2}, \ldots, k_{n}} z_{1}^{k_{1}} z_{2}^{k_{2}} \ldots z_{n}^{k_{n}}=\sum_{|k|=0}^{\infty} a_{k} z^{k}
$$


where $k=\left(k_{1}, k_{2}, \ldots ., k_{n}\right)$ belongs to $\mathscr{N}=\left\{k: k \in \mathbb{C}^{n}\right.$, each $k_{j}$ is rational integer $\}$ and $|k|=k_{1}+k_{2}+\ldots .+k_{n}$.

For $r>(0,0, \ldots, 0)$, the maximum term $\mu(r)=\mu(r, f)$, the maximum modulus $M(r)=M(r, f)$ and the central index $v(r)=v(r, f)=\left(v_{1}(r, f), v_{2}(r, f), \ldots, v_{n}(r, f)\right)$ of entire function $f(z)$ are given by (see [6] and [7]).

$$
\begin{aligned}
& \mu(r)=\mu(r, f)=\max _{k \in \mathscr{N}}\left\{\left|a_{k}\right| r^{k}\right\} \\
& M(r)=M(r, f)=\max _{|z|=r}|f(z)|
\end{aligned}
$$

and

$$
v_{j}(r)=v_{j}(r, f)=\left\{\begin{array}{c}
\max \left[k_{j}:\left|a_{k}\right| r^{k}=\mu(r)\right], \text { if } \mu(r)>0 \\
0, \text { if } \mu(r)=0, \text { for } 1 \leq j \leq n .
\end{array}\right\}
$$

Also, the central index $v(r, f)$ for which maximum term is achieved

$$
|v(r, f)|=v_{1}(r, f)+v_{2}(r, f)+\ldots+v_{n}(r, f)
$$

Definition 1. ([3], p.339) The order $\rho_{f}$ and lower order $\lambda_{f}$ of an entire function $f(z)=f\left(z_{1}, z_{2}, \ldots . ., z_{n}\right)$ are defined as follows

$$
\rho_{f}=\limsup _{r_{1}, r_{2}, \ldots, r_{n} \rightarrow \infty} \frac{\log ^{[2]} M\left(r_{1}, r_{2}, \ldots, r_{n}, f\right)}{\log \left(r_{1} r_{2} \ldots r_{n}\right)}
$$

and

$$
\lambda_{f}=\liminf _{r_{1}, r_{2}, \ldots, r_{n} \rightarrow \infty} \frac{\log ^{[2]} M\left(r_{1}, r_{2}, \ldots, r_{n}, f\right)}{\log \left(r_{1} r_{2} \ldots r_{n}\right)} .
$$

where

$$
\log ^{[k]} x=\log \left(\log ^{[k-1]} x\right) \text { for } k=1,2,3, \ldots \text { and } \log ^{[0]} x=x .
$$

Following Datta and Mallik (see [2]) definitions of hyper order (hyper lower order), generalized order (generalized lower order) of entire functions of two complex variables, we may give the same for the entire functions of $n$-complex variables.

Definition 2. The hyper order $\bar{\rho}_{f}$ and the hyper lower order $\bar{\lambda}_{f}$ of an entire function $f$ are defined as follows:

$$
\bar{\rho}_{f}=\limsup _{r_{1}, r_{2}, \ldots, r_{n} \rightarrow \infty} \frac{\log ^{[3]} M\left(r_{1}, r_{2}, \ldots, r_{n}, f\right)}{\log \left(r_{1} r_{2} \ldots r_{n}\right)}
$$

and

$$
\bar{\lambda}_{f}=\liminf _{r_{1}, r_{2}, \ldots, r_{n} \rightarrow \infty} \frac{\log ^{[3]} M\left(r_{1}, r_{2}, \ldots, r_{n}, f\right)}{\log \left(r_{1} r_{2} \ldots r_{n}\right)} .
$$

Definition 3. Let $l$ be an integer $\geq 1$. The generalized order $\rho_{f}^{[l]}$ and the generalized lower order $\lambda_{f}^{[l]}$ of an entire function $f$ are defined as follows:

$$
\rho_{f}^{[l]}=\limsup _{r_{1}, r_{2}, \ldots, r_{n} \rightarrow \infty} \frac{\log ^{[l+1]} M\left(r_{1}, r_{2}, \ldots, r_{n}, f\right)}{\log \left(r_{1} r_{2} \ldots r_{n}\right)}
$$

and

$$
\lambda_{f}^{[l]}=\liminf _{r_{1}, r_{2}, \ldots, r_{n} \rightarrow \infty} \frac{\log ^{[l+1]} M\left(r_{1}, r_{2}, \ldots, r_{n}, f\right)}{\log \left(r_{1} r_{2} \ldots r_{n}\right)} .
$$

When $l=1$, Definition 3 coincides with Definition 1 and when $l=2$, Definition 3 coincides with Definition 2. In 1988, He and Xiao [5] define the order of an entire function in terms of its central index as follows: 
Definition 4. The order $\rho_{f}$ of an entire function $f(z)$ is defined by

$$
\rho_{f}=\limsup _{r \rightarrow \infty} \frac{\log v(r, f)}{\log r} .
$$

Similarly, the lower order $\lambda_{f}$ of of an entire function $f(z)$ is defined as

$$
\lambda_{f}=\liminf _{r \rightarrow \infty} \frac{\log v(r, f)}{\log r} .
$$

Later in 1999, Chen and Yang [1] define the hyper order of an entire function in terms of the central index in the following manner.

Definition 5. The hyper order $\bar{\rho}_{f}$ of an entire function $f(z)$ is defined by

$$
\bar{\rho}_{f}=\limsup _{r \rightarrow \infty} \frac{\log ^{[2]} v(r, f)}{\log r} .
$$

Similarly, the hyper lower order $\bar{\lambda}_{f}$ of of an entire function $f$ is defined as

$$
\bar{\lambda}_{f}=\liminf _{r \rightarrow \infty} \frac{\log ^{[2]} v(r, f)}{\log r} .
$$

So it is interesting to investigate that whether or not the generalized order of an entire function of several complex variables can be define in terms of its central index.

In this paper, we establish that the generalized order (generalized lower order) of an entire function of several complex variables can be defined in terms of its central index. Also we study some comparative growth measure of composite entire function of several complex variables with respect to left (right) factor of the composite entire function based on their central index.

\section{Lemmas}

In this section we present some lemmas which will be needed in the sequel.

Lemma 1. [6] Let $p, r \in|\Omega|$ and let $\mu(p)$ and $\mu(r)$ be both positive. Then the line integral,

$$
I=\int_{p}^{r} \sum_{j=1}^{n} \frac{v_{j}(x)}{x_{j}} d x_{j}
$$

taken over any connected polygon in $|\Omega|$ with sides parallel to the axes and from $p$ to $r$,

(i) exists,

(ii) is independent of the polygon and

(iii) is such that $\log \mu(r)=\log \mu(p)+I$.

Lemma 2. [6] Let $r \in|\Omega|$. Let $p \in\left|C^{n}\right|$ and be such that $p>>(1,1, \ldots, 1)$, while $p r=\left(p_{1} r_{1}, p_{2} r_{2}, \ldots, p_{n} r_{n}\right)$ still $\in|\Omega|$. Let

$$
N_{j}=\max _{r \leq t \leq p r} v_{j}(t) \text { for } 1 \leq j \leq n
$$

Then 
(i) $\mu(r) \leq M(r) \leq \mu(r) \prod_{j=1}^{n}\left[N_{j}+\frac{p_{j}}{p_{j}-1}\right]$,

(ii) $\mu(r)=M(r)$, if and only if the series $\sum_{|k|=0}^{\infty} a_{k} r^{k}$ has at most one non vanishing term,

(iii) the last relation in (i) is an equality if and only if $\mu(r)=0$.

Lemma 3. Let $f(z)$ be an entire function of $n$-complex variables with generalized order $\rho_{f}^{[l]}$, where l be a positive integer $\geq 1$. Then

$$
\rho_{f}^{[l]}=\limsup _{r_{1}, r_{2}, \ldots, r_{n} \rightarrow \infty} \frac{\log { }^{[l]}\left|v\left(r_{1}, r_{2}, \ldots, r_{n}, f\right)\right|}{\log \left(r_{1} r_{2} \ldots r_{n}\right)} .
$$

Proof. Set

$$
f(z)=\sum_{k=(0,0, \ldots, 0)}^{\infty} a_{k_{1}, k_{2}, \ldots \ldots, k_{n}} z_{1}^{k_{1}} z_{2}^{k_{2}} \ldots z_{n}^{k_{n}}=\sum_{|k|=0}^{\infty} a_{k} z^{k}
$$

By Lemma 1, we see the maximum term $\mu(r)$ of $f$ satisfies

$$
\log \mu(r)=\log \mu(p)+\int_{p}^{r} \sum_{j=1}^{n} \frac{v_{j}(x)}{x_{j}} d x_{j}
$$

Krishna ([6], Corollary 2.9) proved that $v_{j}(r)$ is increasing and right continuous in $j$-th variable for $1 \leq j \leq n$. Therefore, for any $p, r \in|\Omega|$ such that $\mu(r)>0$ and $p>>(1,1, \ldots, 1)$, we get for $1 \leq j \leq n$,

$$
v_{j}(r) \leq \frac{1}{\log p_{j}} \int_{p}^{r} v_{j}\left(r_{1}, \ldots, r_{j-1}, \ldots, r_{n}\right) \frac{d x_{j}}{x_{j}} .
$$

From (1) and (2), we get

$$
\log \mu(r) \geq \log \mu(p)+\sum_{j=1}^{n} v_{j}(r) \log p_{j}
$$

By Lemma 2, we have

$$
\mu(r, f) \leq M(r, f)
$$

It follows from (3) and (4) that

$$
\sum_{j=1}^{n} v_{j}(r) \log p_{j} \leq \log M(r, f)+C_{1}
$$

As $p>>(1,1, \ldots, 1)$ i.e., $p=\left(p_{1}, p_{2}, \ldots, p_{n}\right)>>(1,1, \ldots, 1)$, choosing $p_{j}=2$ for $1 \leq j \leq n$, we get

$$
\begin{aligned}
& \sum_{j=1}^{n} v_{j}(r) \log 2 \leq \log M(r, f)+C_{1} \\
& \Rightarrow \log ^{[l]}|v(r, f)|+\log ^{[l+2]} 2 \leq \log ^{[l+2]} M(r, f)+C_{2} \\
& \Rightarrow \log { }^{[l]}|v(r, f)|+\log ^{[l+1]} 2 \leq \log ^{[l+1]} M(r, f)+C_{2}
\end{aligned}
$$

where $C_{j}(>0)(j=1,2)$ is a suitable constant.

By (6) and Definition 3, we have

$$
\limsup _{r_{1}, r_{2}, \ldots, r_{n} \rightarrow \infty} \frac{\log [l]\left|v\left(r_{1}, r_{2}, \ldots, r_{n}, f\right)\right|}{\log \left(r_{1} r_{2} \ldots r_{n}\right)} \leq \limsup _{r_{1}, r_{2}, \ldots, r_{n} \rightarrow \infty} \frac{\log { }^{[l+1]} M\left(r_{1}, r_{2}, \ldots, r_{n}, f\right)}{\log \left(r_{1} r_{2} \ldots r_{n}\right)}=\rho_{f}^{[l]} .
$$


On the other hand, by choosing $p_{j}=2$ for $1 \leq j \leq n$ i.e., $p=(2,2, \ldots, 2)$ in (i) of Lemma 2 , we have

$$
M(r, f) \leq \mu(r, f) \prod_{j=1}^{n}\left[N_{j}+2\right],
$$

where $N_{j}=\max _{r \leq t \leq p r} v_{j}(t)$, for $1 \leq j \leq n$.

$$
\Rightarrow M(r, f) \leq\left|a_{v(r, f)}\right| r^{v(r, f)} \prod_{j=1}^{n}\left[N_{j}+2\right]
$$

Since $\left\{\left|a_{k}\right|\right\}$ is bounded, from (8) we get

$$
\begin{aligned}
\log M(r, f) & \leq \sum_{j=1}^{n} v_{j}(r) \log r_{j}+\sum_{j=1}^{n} \log N_{j}+C_{3} \\
& \leq \sum_{j=1}^{n}|v(r, f)| \log r_{j}+\sum_{j=1}^{n} \log N_{j}+C_{3} \\
& \leq|v(r, f)| \log \left(r_{1} r_{2} \ldots r_{n}\right)+\log \left(N_{1} N_{2} \ldots N_{n}\right)+C_{3} \\
& \Rightarrow \log ^{[l+1]} M(r, f) \leq \log ^{[l]}|v(r, f)|+\log ^{[l+1]}\left(r_{1} r_{2} \ldots r_{n}\right)+\log ^{[l+1]}\left(N_{1} N_{2} \ldots N_{n}\right)+C_{4}
\end{aligned}
$$

where $C_{j}(>0)(j=3,4)$ are suitable constants. By (9) and Definition 3, we get

$$
\rho_{f}^{[l]}=\limsup _{r_{1}, r_{2}, \ldots, r_{n} \rightarrow \infty} \frac{\log ^{[l+1]} M\left(r_{1}, r_{2}, \ldots, r_{n}, f\right)}{\log \left(r_{1} r_{2} \ldots r_{n}\right)} \leq \limsup _{r_{1}, r_{2}, \ldots, r_{n} \rightarrow \infty} \frac{\log ^{[l]}\left|v\left(r_{1}, r_{2}, \ldots, r_{n}, f\right)\right|}{\log \left(r_{1} r_{2} \ldots r_{n}\right)}
$$

By (7) and (10), Lemma 3 follows.

In the line of Lemma 3, we can prove the following lemma:

Lemma 4. Let $f(z)$ be an entire function of $n$-complex variables with generalized lower order $\lambda_{f}^{[l]}$, where $l$ is a positive integer $\geq 1$. Then

$$
\lambda_{f}^{[l]}=\liminf _{r_{1}, r_{2}, \ldots, r_{n} \rightarrow \infty} \frac{\log [l]\left|v\left(r_{1}, r_{2}, \ldots, r_{n}, f\right)\right|}{\log \left(r_{1} r_{2} \ldots r_{n}\right)} .
$$

The proof is omitted.

\section{Theorems}

In this section we present the main results of the paper.

Theorem 1. Let $f$ and $g$ be two entire functions of $n$-complex variables. Also, let $0<\lambda_{f \circ g}^{[l]} \leq \rho_{f \circ g}^{[l]}<\infty$ and $0<\lambda_{g}^{[l]} \leq$ $\rho_{g}^{[l]}<\infty$. Then

$$
\begin{aligned}
\frac{\lambda_{f \circ g}^{[l]}}{\rho_{g}^{[l]}} & \leq \liminf _{r_{1}, r_{2}, \ldots, r_{n} \rightarrow \infty} \frac{\log ^{[l]}\left|v\left(r_{1}, r_{2}, \ldots, r_{n}, f \circ g\right)\right|}{\log ^{[l]}\left|v\left(r_{1}, r_{2}, \ldots, r_{n}, g\right)\right|} \leq \min \left\{\frac{\lambda_{f \circ g}^{[l]}}{\lambda_{g}^{[l]}}, \frac{\rho_{f \circ g}^{[l]}}{\rho_{g}^{[l]}}\right\} \\
& \leq \max \left\{\frac{\lambda_{f \circ g}^{[l]}}{\lambda_{g}^{[l]}}, \frac{\rho_{f \circ g}^{[l]}}{\rho_{g}^{[l]}}\right\} \leq \limsup _{r_{1}, r_{2}, \ldots, r_{n} \rightarrow \infty} \frac{\log ^{[l]}\left|v\left(r_{1}, r_{2}, \ldots, r_{n}, f \circ g\right)\right|}{\log ^{[l]}\left|v\left(r_{1}, r_{2}, \ldots, r_{n}, g\right)\right|} \leq \frac{\rho_{f \circ g}^{[l]}}{\lambda_{g}^{[l]}}
\end{aligned}
$$


Proof. Using respectively Lemma 3 and Lemma 4 for the entire function $g$, we have for arbitrary positive $\varepsilon$ and for all sufficiently large values of $r_{1}, r_{2}, \ldots, r_{n}$ that

$$
\log ^{[l]}\left|v\left(r_{1}, r_{2}, \ldots, r_{n}, g\right)\right| \leq\left(\rho_{g}^{[l]}+\varepsilon\right) \log \left(r_{1} r_{2} \ldots r_{n}\right)
$$

and

$$
\log ^{[l]}\left|v\left(r_{1}, r_{2}, \ldots, r_{n}, g\right)\right| \geq\left(\lambda_{g}^{[l]}-\varepsilon\right) \log \left(r_{1} r_{2} \ldots r_{n}\right) .
$$

Also, for a sequence of values of each of $r_{1}, r_{2}, \ldots, r_{n}$ tending to infinity

$$
\log ^{[l]}\left|v\left(r_{1}, r_{2}, \ldots, r_{n}, g\right)\right| \leq\left(\lambda_{g}^{[l]}+\varepsilon\right) \log \left(r_{1} r_{2} \ldots r_{n}\right)
$$

and

$$
\log ^{[l]}\left|v\left(r_{1}, r_{2}, \ldots, r_{n}, g\right)\right| \geq\left(\rho_{g}^{[l]}-\varepsilon\right) \log \left(r_{1} r_{2} \ldots r_{n}\right)
$$

Using respectively Lemma 3 and Lemma 4 for the composite entire function $f \circ g$, we have for arbitrary positive $\varepsilon$ and for all sufficiently large values of $r_{1}, r_{2}, \ldots, r_{n}$ that

$$
\log ^{[l]}\left|v\left(r_{1}, r_{2}, \ldots, r_{n}, f \circ g\right)\right| \leq\left(\rho_{f \circ g}^{[l]}+\varepsilon\right) \log \left(r_{1} r_{2} \ldots r_{n}\right)
$$

and

$$
\log ^{[l]}\left|v\left(r_{1}, r_{2}, \ldots, r_{n}, f \circ g\right)\right| \geq\left(\lambda_{f \circ g}^{[l]}-\varepsilon\right) \log \left(r_{1} r_{2} \ldots r_{n}\right)
$$

Again, for a sequence of values of each of $r_{1}, r_{2}, \ldots, r_{n}$ tending to infinity

$$
\log ^{[l]}\left|v\left(r_{1}, r_{2}, \ldots, r_{n}, f \circ g\right)\right| \leq\left(\lambda_{f \circ g}^{[l]}+\varepsilon\right) \log \left(r_{1} r_{2} \ldots r_{n}\right)
$$

and

$$
\log ^{[l]}\left|v\left(r_{1}, r_{2}, \ldots, r_{n}, f \circ g\right)\right| \geq\left(\rho_{f \circ g}^{[l]}-\varepsilon\right) \log \left(r_{1} r_{2} \ldots r_{n}\right) .
$$

Now from (11) and (16), it follows for all sufficiently large values of $r_{1}, r_{2} \ldots, r_{n}$ that

$$
\frac{\log ^{[l]}\left|v\left(r_{1}, r_{2}, \ldots, r_{n}, f \circ g\right)\right|}{\log ^{[l]}\left|v\left(r_{1}, r_{2}, \ldots, r_{n}, g\right)\right|} \geq \frac{\lambda_{f \circ g}^{[l]}-\varepsilon}{\rho_{g}^{[l]}+\varepsilon} .
$$

As $\varepsilon(>0)$ is arbitrary, we obtain

$$
\liminf _{r_{1}, r_{2}, \ldots, r_{n} \rightarrow \infty} \frac{\log ^{[l]}\left|v\left(r_{1}, r_{2}, \ldots, r_{n}, f \circ g\right)\right|}{\log ^{[l]}\left|v\left(r_{1}, r_{2}, \ldots, r_{n}, g\right)\right|} \geq \frac{\lambda_{f \circ g}^{[l]}}{\rho_{g}^{[l]}}
$$

Again, combining (12) and (17), we get for a sequence of values of each of $r_{1}, r_{2}, \ldots, r_{n}$ tending to infinity

$$
\frac{\log ^{[l]}\left|v\left(r_{1}, r_{2}, \ldots, r_{n}, f \circ g\right)\right|}{\log ^{[l]}\left|v\left(r_{1}, r_{2}, \ldots, r_{n}, g\right)\right|} \leq \frac{\lambda_{f \circ g}^{[l]}+\varepsilon}{\lambda_{g}^{[l]}-\varepsilon}
$$

Since $\varepsilon(>0)$ is arbitrary, it follows that

$$
\liminf _{r_{1}, r_{2}, \ldots, r_{n} \rightarrow \infty} \frac{\log ^{[l]}\left|v\left(r_{1}, r_{2}, \ldots, r_{n}, f \circ g\right)\right|}{\log ^{[l]}\left|v\left(r_{1}, r_{2}, \ldots, r_{n}, g\right)\right|} \leq \frac{\lambda_{f \circ g}^{[l]}}{\lambda_{g}^{[l]}}
$$


Similarly, from (14) and (15) it follows for a sequence of values of each of $r_{1}, r_{2}, \ldots, r_{n}$ tending to infinity

$$
\frac{\log ^{[l]}\left|v\left(r_{1}, r_{2}, \ldots, r_{n}, f \circ g\right)\right|}{\log ^{[l]}\left|v\left(r_{1}, r_{2}, \ldots, r_{n}, g\right)\right|} \leq \frac{\rho_{f \circ g}^{[l]}+\varepsilon}{\rho_{g}^{[l]}-\varepsilon} .
$$

As $\varepsilon(>0)$ is arbitrary, we obtain

$$
\liminf _{r_{1}, r_{2}, \ldots, r_{n} \rightarrow \infty} \frac{\log ^{[l]}\left|v\left(r_{1}, r_{2}, \ldots, r_{n}, f \circ g\right)\right|}{\log ^{[l]}\left|v\left(r_{1}, r_{2}, \ldots, r_{n}, g\right)\right|} \leq \frac{\rho_{f \circ g}^{[l]}}{\rho_{g}^{[l]}} .
$$

Now combining (19), (20) and (21), we get

$$
\frac{\lambda_{f \circ g}^{[l]}}{\rho_{g}^{[l]}} \leq \liminf _{r_{1}, r_{2}, \ldots, r_{n} \rightarrow \infty} \frac{\log ^{[l]}\left|v\left(r_{1}, r_{2}, \ldots, r_{n}, f \circ g\right)\right|}{\log ^{[l]}\left|v\left(r_{1}, r_{2}, \ldots, r_{n}, g\right)\right|} \leq \min \left\{\frac{\lambda_{f \circ g}^{[l]}}{\lambda_{g}^{[l]}}, \frac{\rho_{f \circ g}^{[l]}}{\rho_{g}^{[l]}}\right\} .
$$

Now, from (13) and (16) we obtain for a sequence of values of each of $r_{1}, r_{2}, \ldots, r_{n}$ tending to infinity that

$$
\frac{\log ^{[l]}\left|v\left(r_{1}, r_{2}, \ldots, r_{n}, f \circ g\right)\right|}{\log ^{[l]}\left|v\left(r_{1}, r_{2}, \ldots, r_{n}, g\right)\right|} \geq \frac{\lambda_{f \circ g}^{[l]}-\varepsilon}{\lambda_{g}^{[l]}+\varepsilon}
$$

Choosing $\varepsilon \rightarrow 0$ we get that

$$
\limsup _{r_{1}, r_{2}, \ldots, r_{n} \rightarrow \infty} \frac{\log ^{[l]}\left|v\left(r_{1}, r_{2}, \ldots, r_{n}, f \circ g\right)\right|}{\log ^{[l]}\left|v\left(r_{1}, r_{2}, \ldots, r_{n}, g\right)\right|} \geq \frac{\lambda_{f \circ g}^{[l]}}{\lambda_{g}^{[l]}} .
$$

Again from (12) and (15), it follows for all sufficiently large values of $r_{1}, r_{2}, \ldots, r_{n}$

$$
\frac{\log ^{[l]}\left|v\left(r_{1}, r_{2}, \ldots, r_{n}, f \circ g\right)\right|}{\log ^{[l]}\left|v\left(r_{1}, r_{2}, \ldots, r_{n}, g\right)\right|} \leq \frac{\rho_{f \circ g}^{[l]}+\varepsilon}{\lambda_{g}^{[l]}-\varepsilon} .
$$

As $\varepsilon(>0)$ is arbitrary, we obtain

$$
\limsup _{r_{1}, r_{2}, \ldots, r_{n} \rightarrow \infty} \frac{\log ^{[l]}\left|v\left(r_{1}, r_{2}, \ldots, r_{n}, f \circ g\right)\right|}{\log ^{[l]}\left|v\left(r_{1}, r_{2}, \ldots, r_{n}, g\right)\right|} \leq \frac{\rho_{f \circ g}^{[l]}}{\lambda_{g}^{[l]}} .
$$

Similarly, combining (11) and (18), we get for a sequence of values of each of $r_{1}, r_{2}, \ldots, r_{n}$ tending to infinity

$$
\frac{\log ^{[l]}\left|v\left(r_{1}, r_{2}, \ldots, r_{n}, f \circ g\right)\right|}{\log ^{[l]}\left|v\left(r_{1}, r_{2}, \ldots, r_{n}, g\right)\right|} \geq \frac{\rho_{f \circ g}^{[l]}-\varepsilon}{\rho_{g}^{[l]}+\varepsilon} .
$$

Since $\varepsilon(>0)$ is arbitrary, it follows

$$
\limsup _{r_{1}, r_{2}, \ldots, r_{n} \rightarrow \infty} \frac{\log ^{[l]}\left|v\left(r_{1}, r_{2}, \ldots, r_{n}, f \circ g\right)\right|}{\log ^{[l]}\left|v\left(r_{1}, r_{2}, \ldots, r_{n}, g\right)\right|} \geq \frac{\rho_{f \circ g}^{[l]}}{\rho_{g}^{[l]}} .
$$


Therefore, combining (23), (24) and (25) we get

$$
\max \left\{\frac{\lambda_{f \circ g}^{[l]}}{\lambda_{g}^{[l]}}, \frac{\rho_{f \circ g}^{[l]}}{\rho_{g}^{[l]}}\right\} \leq \limsup _{r_{1}, r_{2}, \ldots, r_{n} \rightarrow \infty} \frac{\log ^{[l]}\left|v\left(r_{1}, r_{2}, \ldots, r_{n}, f \circ g\right)\right|}{\log ^{[l]}\left|v\left(r_{1}, r_{2}, \ldots, r_{n}, g\right)\right|} \leq \frac{\rho_{f \circ g}^{[l]}}{\lambda_{g}^{[l]}} .
$$

Thus the theorem follows from (22) and (26).

Remark. If we take $0<\lambda_{f}^{[l]} \leq \rho_{f}^{[l]}<\infty$ instead of $0<\lambda_{g}^{[l]} \leq \rho_{g}^{[l]}<\infty$ and the other conditions remain the same, then also Theorem 1 holds with $g$ replaced by $f$ in the denominator as we see in the next theorem.

Theorem 2. Let $f$ and $g$ be two entire functions of $n$-complex variables. Also let $0<\lambda_{f \circ g}^{[l]} \leq \rho_{f \circ g}^{[l]}<\infty$ and $0<\lambda_{f}^{[l]} \leq$ $\rho_{f}^{[l]}<\infty$. Then

$$
\begin{aligned}
\frac{\lambda_{f \circ g}^{[l]}}{\rho_{f}^{[l]}} & \leq \liminf _{r_{1}, r_{2}, \ldots, r_{n} \rightarrow \infty} \frac{\log ^{[l]}\left|v\left(r_{1}, r_{2}, \ldots, r_{n}, f \circ g\right)\right|}{\log ^{[l]}\left|v\left(r_{1}, r_{2}, \ldots, r_{n}, f\right)\right|} \leq \min \left\{\frac{\lambda_{f \circ g}^{[l]}}{\lambda_{f}^{[l]}}, \frac{\rho_{f \circ g}^{[l]}}{\rho_{f}^{[l]}}\right\} \\
& \leq \max \left\{\frac{\lambda_{f \circ g}^{[l]}}{\lambda_{f}^{[l]}}, \frac{\rho_{f \circ g}^{[l]}}{\rho_{f}^{[l]}}\right\} \leq \limsup _{r_{1}, r_{2}, \ldots, r_{n} \rightarrow \infty} \frac{\log ^{[l]}\left|v\left(r_{1}, r_{2}, \ldots, r_{n}, f \circ g\right)\right|}{\log ^{[l]}\left|v\left(r_{1}, r_{2}, \ldots, r_{n}, f\right)\right|} \leq \frac{\rho_{f \circ g}^{[l]}}{\lambda_{f}^{[l]}}
\end{aligned}
$$

The proof is omitted.

Example 1. Taking $f=\exp z, g=z$ and $n=1$ one can easily verify that the sign " $\leq$ " in Theorem 2 cannot be replaced by " $<$ " only.

Corollary 1. Let $f$ and $g$ be two entire functions of $n$-complex variables such that $0<\lambda_{f \circ g}^{[l]} \leq \rho_{f \circ g}^{[l]}<\infty$. Also let $0<\lambda_{f}^{[l]}=$ $\rho_{f}^{[l]}<\infty$. Then

$$
\liminf _{r_{1}, r_{2}, \ldots, r_{n} \rightarrow \infty} \frac{\log ^{[l]}\left|v\left(r_{1}, r_{2}, \ldots, r_{n}, f \circ g\right)\right|}{\log ^{[l]}\left|v\left(r_{1}, r_{2}, \ldots, r_{n}, f\right)\right|}=\frac{\lambda_{f \circ g}^{[l]}}{\lambda_{f}^{[l]}}
$$

and

$$
\limsup _{r_{1}, r_{2}, \ldots, r_{n} \rightarrow \infty} \frac{\log ^{[l]}\left|v\left(r_{1}, r_{2}, \ldots, r_{n}, f \circ g\right)\right|}{\log ^{[l]}\left|v\left(r_{1}, r_{2}, \ldots, r_{n}, f\right)\right|}=\frac{\rho_{f \circ g}^{[l]}}{\rho_{f}^{[l]}}
$$

\section{Conclusion}

The main aim of the paper is to investigate some growth properties of entire function of several complex variables on the basis of central index. There are several other growth properties of entire function of several complex variables, namely type(weak type), exponent of convergence, L-order(lower order), L*-order(lower order), and properties related to these can be investigate using the central index and we feel that our theory will provide a helping tool for the investigation.

\section{Acknowledgement}

This research work is supported by the Council of Scientific and Industrial Research, Extra Mural Research Division, CSIR Complex, Library Avenue, Pusa, New Delhi-110012, India, Under the sanctioned file no. 09/285(0069)/2016-EMR-I.

\section{Competing interests}

The authors declare that they have no competing interests. 


\section{Authors' contributions}

All authors have contributed to all parts of the article. All authors read and approved the final manuscript.

\section{References}

[1] Chen, Z. X. and Yang, C. C.: Some further results on the zeros and growths of entire solutions of second order linear differential equations, Kodai Math J., 22(1999), pp. 273-285.

[2] Datta S. K. and Mallik, M.: Growth properties of composite entire functions of two complex variables, Int. J. Contemp. Math. Sciences, Vol.6, No. 24(2011), pp.1201-1212.

[3] Fucks, B. A.: Introduction to the theory of functions of several complex variables, Amer. Math. Soc., 1963.

[4] Filevych, P. V.: On the growth of the maximum modulus of an entire function depending on the growth of its central index, Ufa Mathematical Journal, Vol.3, No.1(2011), pp.92-100.

[5] He Y.Z. and Xiao X.Z.: Algebroid functions and ordinary differential equations. Science Press, Beijing, 1988.

[6] Krishna, J. G.: Maximum term of a power series in one and several complex variables, Pacific Journal of Mathematics, Vol. 29, No. 3(1969), pp. 609-622.

[7] Krishna, J. G.: Probabilistic techniques leading to a Valiron-type theorem in several complex variables, Ann. Math. Statist., Vol. 41(1970), pp. 2126-2129.

[8] Kumar S. and Srivastava, G. S.: Maximum term and lower order of entire functions of several complex variables, Bulletin of Mathematical Analysis and Applications, Vol. 3, No.1 (2011), pp. 156-164. 